\title{
Designed synthesis of tunable amorphous carbon nanotubes (a-CNTs) by a novel route and their oxidation resistance properties
}

\author{
LONGLONG XU ${ }^{\mathrm{a}}$, YIFU ZHANG ${ }^{\mathrm{a}, \mathrm{b}}$, XIONGZHI ZHANG ${ }^{\mathrm{a}}$, YU HUANG ${ }^{\mathrm{c}}$, XIAOYU TAN ${ }^{\mathrm{b}}$, \\ CHI HUANG ${ }^{\mathrm{a}}$, XIAO MEI $^{\mathrm{d}}$, FEI NIU ${ }^{\mathrm{a}, *}$, CHANGGONG MENG $^{\mathrm{b}}$ and GONGZHEN CHENG ${ }^{\mathrm{a}}$ \\ ${ }^{a}$ College of Chemistry and Molecular Sciences, Wuhan University, Wuhan 430072, China

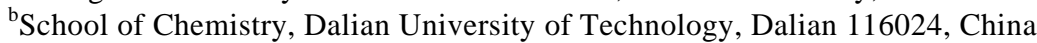 \\ ${ }^{\mathrm{c} S}$ School of Printing and Packaging, Wuhan University, Wuhan 430079, China \\ ${ }^{\mathrm{d}}$ School of Resources and Environmental Science, Wuhan University, Wuhan 430079, China
}

MS received 20 September 2013; revised 30 October 2013

\begin{abstract}
Tunable amorphous carbon nanotubes (a-CNTs) were successfully synthesized using $\mathrm{V}_{3} \mathrm{O}_{7} \cdot \mathrm{H}_{2} \mathrm{O}$ and glucose solution as the starting materials by a novel route for the first time. The as-obtained samples were separately characterized by scanning electron microscopy (SEM), transmission electron microscopy (TEM), $\mathrm{X}$-ray powder diffraction (XRD), energy-dispersive spectrometer (EDS), elemental analysis (EA), Fourier transform infrared spectroscopy (FT-IR) and Raman spectrum. The results showed that the as-obtained a-CNTs had uniform diameters with outer diameter ranging from 140 to $250 \mathrm{~nm}$ and inner diameter about $28 \mathrm{~nm}$ on an average, and their length was up to several micrometres. $\mathrm{No} \mathrm{VO}_{x}$ residues remaining in a-CNTs showed the as-obtained a-CNTs with high purity. The as-prepared a-CNTs were a kind of hydrogenated a-CNTs containing both the $s p^{3}$ - and $s p^{2}$-type carbons. Furthermore, the thermal stability of the as-obtained a-CNTs in the air atmosphere were investigated by thermo-gravimetric/differential thermal analyser (TG-DTA), revealing that the as-obtained a-CNTs had good thermal stability and oxidation resistance below $300{ }^{\circ} \mathrm{C}$ in air.
\end{abstract}

Keywords. Amorphous carbon nanotubes; chemical synthesis; structures; oxidation resistance properties.

\section{Introduction}

Since the discovery of carbon nanotubes (CNTs) in 1991 (Iijima 1991), CNTs have promptly attracted much attention owing to their extraordinary mechanical, chemical and electrical properties as well as their potential applications (Lehman et al 2011; Ionescu et al 2012; Nanot et al 2012). Previous works were mostly focused on either multi-walled CNTs or single-walled CNTs. In recent years, amorphous carbon nanotubes (a-CNTs) have attracted increasing attention because of their amorphous structure being different from crystalline carbon nanotubes. The walls of a-CNTs are composed of many carbon clusters, whose characteristic is of short-distance order and long-distance disorder (Zhao et al 2006). The properties of a-CNTs are different from single-walled and multi-walled CNTs (Nishino et al 2003; Zhao et al 2006, 2009; Lonappan et al 2011; Song et al 2011). The a-CNTs are favourable for certain applications such as in some nanoelectronics and sensor devices (Zhao et al 2009). Thus, a-CNTs become another focus of research. So far,

\footnotetext{
*Author for correspondence (feiniu@whu.edu.cn)
}

some methods including CVD, arc discharge, anodic aluminium oxide (AAO) template, solvothermal method, etc., have been developed to synthesize a-CNTs (Ci et al 2001, 2003; Nishino et al 2003; Zhao et al 2005, 2006, 2009; Chen et al 2006; Luo et al 2006; Liu et al 2004, 2007a,b). However, requirements of high synthesis temperature and pressure, poisonous reagents, catalyst supports, complicated processing steps, longer synthesis period or expensive costs may still be the major drawbacks arising from the above-mentioned techniques (Tan et al 2012). Therefore, it is necessary and meaningful to explore new processes for the preparation of a-CNTs, which could overcome the disadvantages of these techniques, and is crucially important to the future application of a-CNTs. In our previous report, a-CNTs were synthesized using vanadium dioxide as the template (Zhang et al 2013c). Herein, we have designed and developed a novel route for the synthesis of a-CNTs using $\mathrm{V}_{3} \mathrm{O}_{7} \cdot \mathrm{H}_{2} \mathrm{O}$ and glucose solution as the starting materials. The as-obtained a-CNTs have high purity and a kind of hydrogenated a-CNTs containing both the $s p^{3}$ - and $s p^{2}$ type carbons. Besides, the as-synthesized a-CNTs have good thermal stability and oxidation resistance below $300{ }^{\circ} \mathrm{C}$ in air. 


\section{Experimental}

All reagents used in the experiments were of analytical grade and used without any further purification. The synthetic route for a-CNTs was schematically described as follows

$$
\begin{aligned}
& \mathrm{V}_{2} \mathrm{O}_{5} \frac{\text { EtOH, } \mathrm{H}_{2} \mathrm{O}}{\text { Hydrothermal }} \mathrm{V}_{3} \mathrm{O}_{7} \cdot \mathrm{H}_{2} \mathrm{O} \stackrel{\text { Glucose solution }}{\text { Hydrothermal }} \\
& \mathrm{V}_{3} \mathrm{O}_{7} \cdot \mathrm{H}_{2} \mathrm{O} @ \mathrm{C} \stackrel{\text { Thermal }}{\longrightarrow} \mathrm{VO}_{x} @ \mathrm{C} \stackrel{\text { Hert gas }}{\longrightarrow} \text { a-CNTs. }
\end{aligned}
$$

The synthesis of $\mathrm{V}_{3} \mathrm{O}_{7} \cdot \mathrm{H}_{2} \mathrm{O}$ and $\mathrm{V}_{3} \mathrm{O}_{7} \cdot \mathrm{H}_{2} \mathrm{O} @ \mathrm{C}$ was based on previous reports (Zhang et al 2010, 2011a,c). For the preparation of a-CNTs, $\mathrm{V}_{3} \mathrm{O}_{7} \cdot \mathrm{H}_{2} \mathrm{O} @ \mathrm{C}$ was first heated in a tube furnace with $5{ }^{\circ} \mathrm{C} / \mathrm{min}$ heating rate under a flow of argon (99.999\%) gas at a given temperature for a given time. The purpose of this step is to stabilize the framework of carbon. After the heating treatment, the above products were treated by dilute $\mathrm{HCl}$ solution to remove $\mathrm{VO}_{x}$ in the a-CNTs, and high purity a-CNTs were obtained.

The morphology of the products was observed by the scanning electron microscopy (SEM, Quanta 200) and transmission electron microscopy (TEM, JEM-2100 HR). $\mathrm{X}$-ray powder diffraction (XRD) was carried out on $\mathrm{D} 8 \mathrm{X}$-ray diffractometer equipment with $\mathrm{CuK} \alpha$ radiation, $\lambda=1.54060 \AA$. The elemental analysis (EA) of the sample was carried out using a Vario EL III equipment (Germany). Raman spectrum was taken on an RM-1000 spectrometer (confocal Raman microspectroscopy) with an argon-ion laser at an excitation wavelength of $514.5 \mathrm{~nm}$. Fourier transform infrared spectroscopy (FT-IR) was recorded on a Nicolet 60 -SXB spectrometer from 4000 to $400 \mathrm{~cm}^{-1}$ with a resolution of $4 \mathrm{~cm}^{-1}$. Energy-dispersive

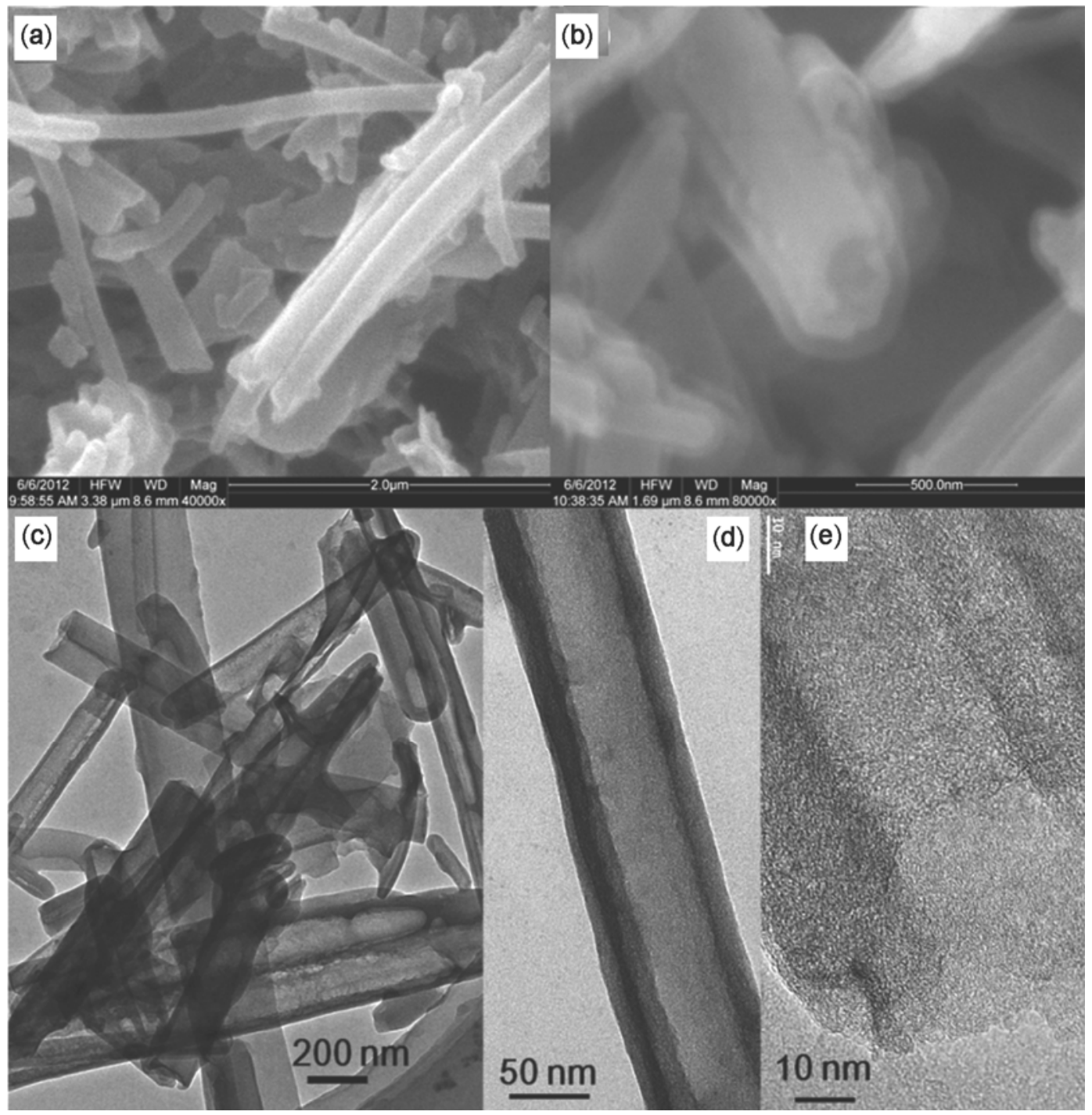

Figure 1. Morphology of the as-obtained a-CNTs: (a and b) SEM images; (c and d) TEM images and (e) HRTEM image. 
spectrometer (EDS) was carried out on the equipment Quanta 200. Thermo-gravimetric/differential thermal analysis (TG/DTA) was performed on SETSYS-1750 (AETARAM Instruments). About $5 \mathrm{mg}$ of the as-obtained sample was heated in an $\mathrm{Al}_{2} \mathrm{O}_{3}$ crucible in air atmosphere from ambient temperature to $600{ }^{\circ} \mathrm{C}$ at a constant rise of temperature $\left(10{ }^{\circ} \mathrm{C} / \mathrm{min}\right)$.

\section{Results and discussion}

The morphology and structure of the as-obtained sample were evaluated by SEM and TEM, as shown in figure 1 . It can be observed that the sample consists of wire-like shape with length up to several micrometres in figure 1(a) and tubular shape with open end is clearly seen in figure 1(b). The TEM images give the clear insight into the core-shell structure of the as-obtained sample, as shown in figure $1(c-f)$. The contrast grade between core and shell also indicates that the sample consists of large-scale nanotubes (figure 1c), in good agreement with the SEM observation. A typical TEM image of one single nanotube is provided in figure $1(\mathrm{~d})$, and the dark area in the image corresponds to the wall of the nanotube. The nanotubes have uniform diameters, with outer diameter ranging from 140 to $250 \mathrm{~nm}$ and inner diameter about $28 \mathrm{~nm}$ on average. The higher magnification TEM image (figure 1f) reveals that the nanotube is completely amorphous, which agrees well with the XRD patterns presented in figure 2 . According to our designed route, the a-CNTs are probably synthesized. Further information about the composition of the amorphous nanotube was collected from the XRD, EA, EDS, FT-IR and Raman tests.

Figure 2(a) shows the XRD pattern of the as-obtained nanotubes. Only a broadened peak ranging from 15 to $25^{\circ}$ can be detected, which reveals that the as-prepared nanotube is amorphous carbon (Zhang et al 2012b,c, 2013a,b). The EDS spectrum (figure 3a) reveals that

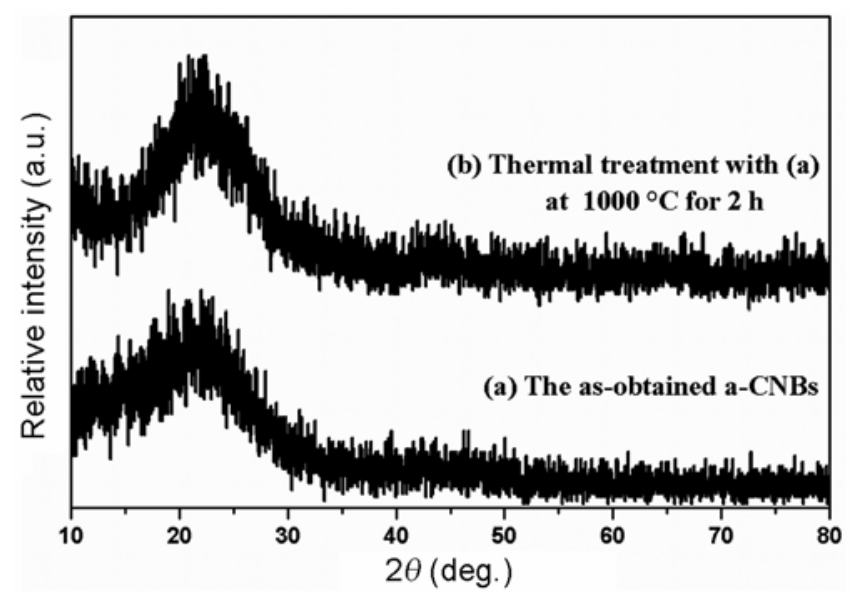

Figure 2. XRD patterns of the as-obtained a-CNTs. the as-synthesized a-CNTs consist of $\mathrm{C}$ and $\mathrm{O}$ elements. Both XRD and EDS manifest that no residual reactant of $\mathrm{VO}_{x}$ remains within the sample.

The result of EA shows that the prepared a-CNTs contain $86.22 \mathrm{wt} \%$ of $\mathrm{C}$ and $2.287 \mathrm{wt} \%$ of $\mathrm{H}$, and the atomic ratio of $\mathrm{C}$ and $\mathrm{H}$ is $3 \cdot 142: 1$. High $\mathrm{C} / \mathrm{H}$ atomic ratio and relatively high $\mathrm{H}$ content indicate that this solid might be a kind of hydrogenated a-CNTs, which can be further
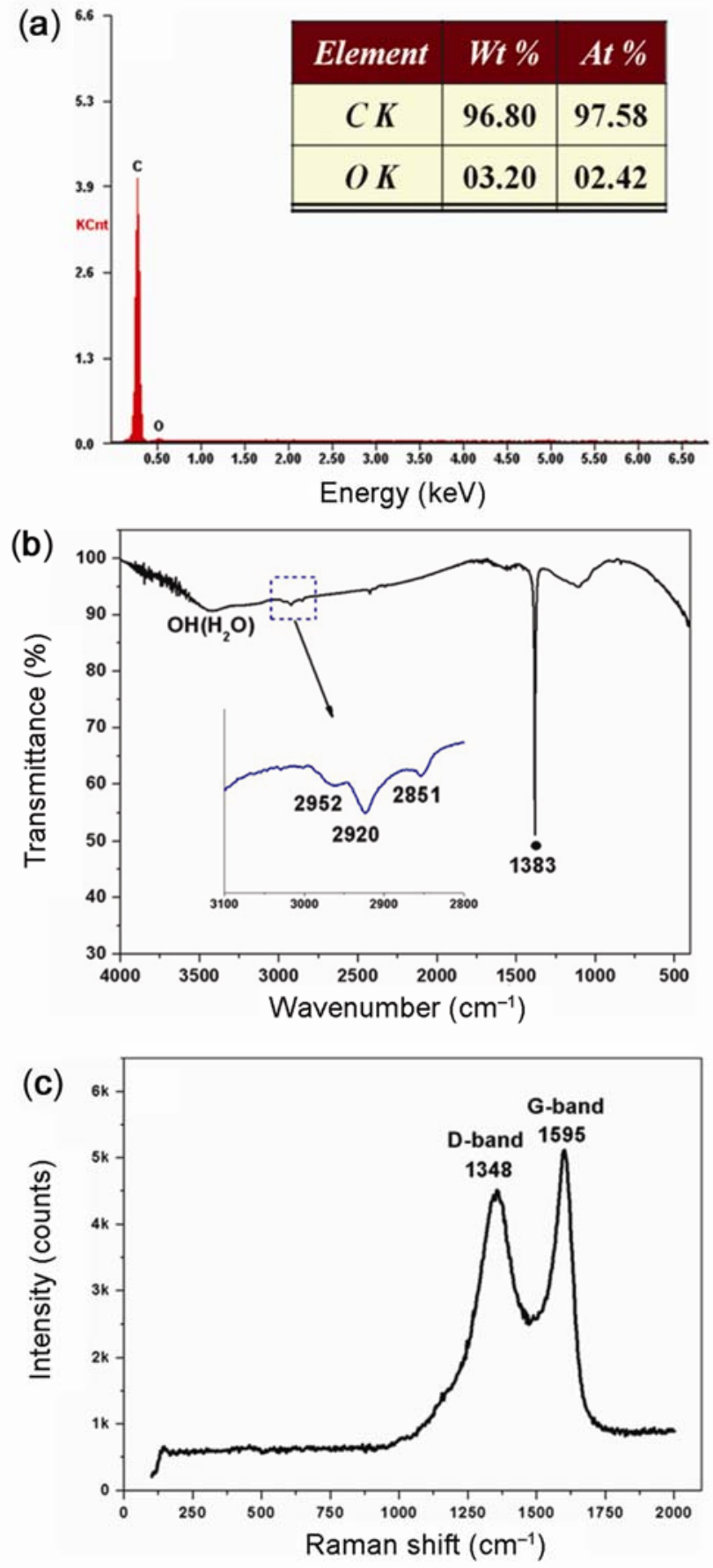

Figure 3. Composition of the as-obtained a-CNTs: (a) EDS spectrum; (b) IR spectrum and (c) Raman spectrum. 
proved by FT-IR. FT-IR spectrum of the as-obtained a-CNTs shows the characteristic $\mathrm{C}-\mathrm{H}$ stretching vibration bands, as depicted in figure 3(b). The absorption bands located at 2851 and $2920 \mathrm{~cm}^{-1}$ can be assigned to $s p^{3}$ $\mathrm{CH}_{2}$-symmetric and (-)asymmetric bands, respectively. The peak at $2952 \mathrm{~cm}^{-1}$ is the characteristic $s p^{2} \mathrm{CH}_{2^{-}}$ symmetric band, although its asymmetric band at about $3080 \mathrm{~cm}^{-1}$ is not observed, because it is a weak absorption band. Besides, the absorptions at 3425 and $1383 \mathrm{~cm}^{-1}$ are due to water and nitrate $\left(\mathrm{NO}_{3}^{-}\right)$adsorbed on the $\mathrm{KBr}$ and can be disregarded (Ros et al 2002; Zhang et al 2012a,f).

Important information of the structure of the asobtained a-CNTs could be provided by Raman spectrum. Figure 3(c) represents a typical Raman spectrum of a-CNTs, which is characteristic for amorphous carbon because of the presence of the D- $\left(1348 \mathrm{~cm}^{-1}\right)$ and G-bands (1595 cm $\mathrm{cm}^{-1}$ ) (Ilie et al 2002; Odani et al 2006). It was reported that the D-band $\left(1348 \mathrm{~cm}^{-1}\right)$ is usually associated with the vibrations of carbon atoms with dangling bonds for the in-plane terminations of disordered graphite, and the G-band (corresponding to the $E_{2 \mathrm{~g}}$ mode) is closely related to the vibration in all $\mathrm{sp}^{2}$-bonded carbon atoms in a two-dimensional hexagonal lattice, such as in a graphitic layer, corresponding to the $E_{2 \mathrm{~g}}$ mode (Dresselhaus et al 1999; llie et al 2002; Odani et al 2006; Sun et al 2006; Zhang et al 2012d,e). These two peaks are broad in figure 3(c), indicating that the carbon in the as-prepared a-CNTs is of poor crystallinity, corresponding to the previous report (Sun and Li 2004; Zhang et al 2011b, 2012b). The intensity ratio of the G- and D-bands was $I_{\mathrm{G}} / I_{\mathrm{D}}=1.15$ for the as-obtained a-CNTs. The relatively high intensity of the D-peak further proves that the as-synthesized a-CNTs comprise disordered carbon. Therefore, Raman spectrum further confirms that the carbon in the as-obtained a-CNTs is disordered, which is well consistent with the HRTEM and XRD observations.

On the basis of the above results, hydrogenated a-CNTs are successfully synthesized at the current conditions, and the as-obtained a-CNTs contain both the $s p^{3}$ - and $s p^{2}$-type carbons. The thickness of the walls of a-CNTs can be changed within a certain range. If the carbon thickness coating on the precursor is higher (Zhang et al 2011a), a thick amorphous carbon wall will be obtained. This kind of a-CNTs is expected to find potential applications since the outer surface of a-CNTs is very reactive toward functionalization by chemical or physical methods due to its amorphous nature. However, the as-obtained a-CNTs cannot be transformed to CNTs at $1000^{\circ} \mathrm{C}$, as shown in figure 2(b).

Therefore, a-CNTs were successfully synthesized using $\mathrm{V}_{3} \mathrm{O}_{7} \cdot \mathrm{H}_{2} \mathrm{O}$ and glucose solution as the starting materials by a novel route. Table 1 lists the synthetic methods of a-CNTs in the literatures and our results. The requirements

Table 1. Summary of the synthetic methods of amorphous carbon nanotubes (a-CNTs) in the literatures.

\begin{tabular}{|c|c|c|c|c|}
\hline Serial & Carbon source & Catalyst & Method & Description and reference \\
\hline 1 & Polytetrafluoroethylene & Ferrous chloride & Thermal under vacuum & $\begin{array}{l}\text { The product has impurity, } \\
\text { containing very minor } \\
\text { amount of Fe and } \mathrm{Cl} \\
\text { (Nishino et al 2003) }\end{array}$ \\
\hline 2 & Citric acid & $\begin{array}{l}\text { Porous anodic aluminium } \\
\text { oxide (AAO) } \\
\text { membrane as template }\end{array}$ & Thermal & Zhao et al (2009) \\
\hline 3 & $\begin{array}{l}\text { Ferrocene and chloride } \\
\text { find powders }\end{array}$ & - & Thermal & Liu et al (2007a) \\
\hline 4 & $\begin{array}{l}\text { Polyacrylonitrile (PAN) } \\
\text { and polystyrene-block- } \\
\text { polyacrylonitrile } \\
\text { (PS-b-PAN) }\end{array}$ & $\begin{array}{l}\text { Porous (AAO) membrane } \\
\text { as template }\end{array}$ & Casting and pyrolysis & Chen et al (2006) \\
\hline 5 & Methane & $\mathrm{Ni} / \mathrm{Al}$ & $\begin{array}{l}\text { Chemical vapour } \\
\text { deposition (CVD) }\end{array}$ & $\begin{array}{l}\text { The product has impurity, } \\
\text { containing a little of Al } \\
\text { and Ni (Zhao et al 2006) }\end{array}$ \\
\hline 6 & Mixed $\mathrm{CH}_{4} / \mathrm{H}_{2}$ & Ni thin film & $\begin{array}{l}\text { Radio frequency plasma- } \\
\text { enhanced CVD }\end{array}$ & Liu et al (2007b) \\
\hline 7 & Benzene and ferrocene & - & CVD & $\begin{array}{l}\text { Benzene solution with a } \\
\text { given content of ferrocene } \\
\text { and a small amount of } \\
\text { thiophene was used } \\
\text { (Ci et al 2001) }\end{array}$ \\
\hline 8 & Graphite powders & Co/Ni alloy & Modified arc discharge & $\begin{array}{l}\text { Precision instrument and } \\
\text { hydrogen gas atmosphere } \\
\text { (Zhao et al 2005) }\end{array}$ \\
\hline 9 & Graphite powders & $\mathrm{FeS}$ & $\begin{array}{l}\text { Temperature-controlled } \\
\text { DC arc discharge }\end{array}$ & Liu et al (2004) \\
\hline 10 & Glucose & Vanadium oxide as template & & Our work \\
\hline
\end{tabular}




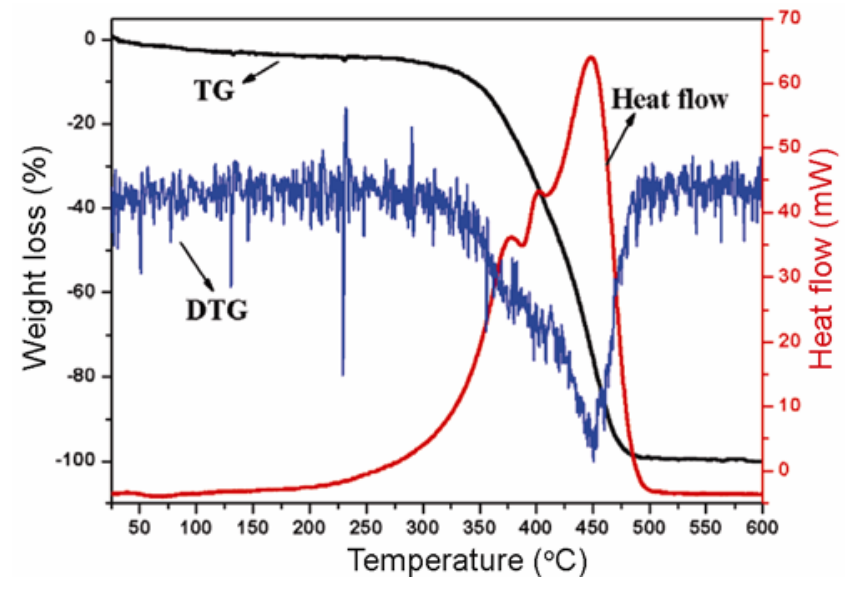

Figure 4. Thermal stability of the as-obtained a-CNTs in the air atmosphere.

of precision instrument, poisonous reagents, catalyst supports, complicated processing steps, longer synthesis period or expensive costs may be the major drawbacks arising from the above-mentioned techniques. To synthesize a-CNTs, we only used glucose (which is very cheap and easily obtained) as the carbon source and vanadium oxide as the template by the hydrothermal route and thermal treatment, which shows our method is very convenient for preparing a-CNTs. Therefore, a novel route was explored to successfully synthesize a-CNTs using $\mathrm{V}_{3} \mathrm{O}_{7} \cdot \mathrm{H}_{2} \mathrm{O}$ and glucose solution as the starting materials.

Furthermore, the thermal stability of the as-obtained a-CNTs in the air atmosphere was investigated by TG-DTA, as shown in figure 4 . The weight loss ca $2 \cdot 4 \%$ below $120^{\circ} \mathrm{C}$ in the TG curve can be due to the release of water absorbed on the surface of a-CNTs. The weight loss starting at about $300{ }^{\circ} \mathrm{C}$ and finishing at about $490{ }^{\circ} \mathrm{C}$ is associated with a-CNTs reacting with $\mathrm{O}_{2}$. There is no residue after the TG test, indicating that the as-obtained a-CNTs have high purity, in agreement with XRD and EDS observations. Both DTG and heat flow curves reveal the fierce oxidation of a-CNTs is located at $450{ }^{\circ} \mathrm{C}$ and the oxidation process finishes at $490^{\circ} \mathrm{C}$. Therefore, the as-obtained a-CNTs have good thermal stability and oxidation resistance below $300{ }^{\circ} \mathrm{C}$ in air.

\section{Conclusions}

In conclusion, a novel route was explored to successfully synthesize a-CNTs using $\mathrm{V}_{3} \mathrm{O}_{7} \cdot \mathrm{H}_{2} \mathrm{O}$ and glucose solution as the starting materials. The as-obtained a-CNTs were separately characterized by SEM, TEM, HRTEM, XRD, EDS, EA, FT-IR and Raman. The as-prepared a-CNTs have uniform diameters, with outer diameter ranging from 140 to $250 \mathrm{~nm}$ and inner diameter about $28 \mathrm{~nm}$ on average, and their length is up to several micrometres. No
$\mathrm{VO}_{x}$ residues remaining in a-CNTs reveal that the as-obtained a-CNTs are of high purity, and the asprepared a-CNTs are a kind of hydrogenated a-CNTs containing both the $s p^{3}$ - and $s p^{2}$-type carbons. The thickness of the walls of a-CNTs can be changed within a certain range. The as-obtained a-CNTs cannot be transformed to CNTs at $1000{ }^{\circ} \mathrm{C}$. Furthermore, the thermal stability of the as-obtained a-CNTs in the air atmosphere was investigated by TG-DTA, revealing that the a-CNTs have good thermal stability and oxidation resistance below $300{ }^{\circ} \mathrm{C}$ in air.

\section{Acknowledgements}

This work was partially supported by the National Natural Science Foundation of China (grant no. 21271037), the Fundamental Research Funds for the Central Universities and China Postdoctoral Science Foundation funded project.

\section{Electronic Supplementary Material}

Supplementary material pertaining to this article is available on the Bulletin of Materials Science website (www.ias.ac.in/matersci).

\section{References}

Chen J T, Shin K, Leiston-Belanger J M, Zhang $\mathrm{M}$ and Russell T P 2006 Adv. Funct. Mater. 161476

Ci L, Wei B, Xu C, Liang J, Wu D, Xie S, Zhou W, Li Y, Liu Z and Tang D 2001 J. Cryst. Growth 233823

Ci L, Zhu H, Wei B, Xu C and Wu D 2003 Appl. Surf. Sci. 20539

Dresselhaus M S, Dresselhaus G and Eklund M A P 1999 In Analytical application of Raman spectroscopy (Oxford: Blackwell Science) Chap. 9

Iijima S 1991 Nature 35456

Ionescu M I, Zhang Y, Li R, Abou-Rachid H and Sun X 2012 Appl. Surf. Sci. 2584563

Lehman J H, Terrones M, Mansfield E, Hurst K E and Meunier V 2011 Carbon 492581

Liu B, Jia D, Zhou Y, Feng H and Meng Q 2007a Carbon 45 1710

Liu J W, Wang X, Zheng W T, Li J X, Guan Q F, Su Y D, Qi J L and Jiang Q 2007b Carbon 45681

Liu Y, Xiaolong S, Tingkai Z, Jiewu Z, Hirscher M and Philipp F 2004 Carbon 421852

Llie A, Durkan C, Milne W I and Welland M E 2002 Phys. Rev. B66 045412/1

Lonappan L, Issac S, Joseph R, Thomas D and Kumar K G 2011 Micro Nano Lett. 6867

Luo T, Chen L, Bao K, Yu W and Qian Y 2006 Carbon 44 2844

Nanot S, Hároz E H, Kim J-H, Hauge R H and Kono J 2012 Adv. Mater. 244977

Nishino H, Yamaguchi C, Nakaoka H and Nishida R 2003 Carbon 412165 
Odani A, Pol V G, Pol S V, Koltypin M, Gedanken A and Aurbach D 2006 Adv. Mater. 181431

Ros T G, van Dillen A J, Geus J W and Koningsberger D C 2002 Chem.-Eur. J. 81151

Song X, Yang F, Wang X and Zhang K 2011 Micro. Nano Lett. 6827

Sun X and Li Y 2004 Angew. Chem. Int. Ed. 43597

Sun X M, Liu J F and Li Y D 2006 Chem. Mater. 18 3486

Tan K H, Ahmad R, Leo B F, Yew M C, Ang B C and Johan M R 2012 Mater. Res. Bull. 471849

Zhang Y, Liu X, Xie G, Yu L, Yi S, Hu M and Huang C 2010 Mater. Sci. Eng. B175 164

Zhang Y, Liu X, Chen D, Yu L, Nie J, Yi S, Li H and Huang C 2011a J. Alloys Compd. 509 L69

Zhang Y, Liu X, Nie J, Yu L, Zhong Y and Huang C 2011b J. Solid State Chem. 184387

Zhang Y, Zhou M, Fan M, Huang C, Chen C, Cao Y, Li H and Liu X 2011c Curr. Appl. Phys. 111159

Zhang Y, Fan M, Hu L, Wu W, Zhang J, Liu X, Zhong Y and Huang C 2012a Appl. Surf. Sci. 2589660
Zhang Y, Fan M, Liu X, Huang C and Li H 2012b Eur. J. Inorg. Chem. 20121650

Zhang Y, Fan M, Wu W, Hu L, Zhang J, Mao Y, Huang C and Liu X 2012c Mater. Lett. 71127

Zhang Y, Zhang F, Yu L, Fan M, Zhong Y, Liu X, Mao Y and Huang C 2012d Colloids Surf. A396 144

Zhang Y, Zhang J, Nie J, Zhong Y, Liu X and Huang C 2012e Micro Nano Lett. 7782

Zhang Y, Zhang J, Zhong Y, Yu L, Deng Y, Huang C and Liu X 2012f Appl. Surf. Sci. 263124

Zhang Y, Chen C, Zhang J, Hu L, Wu W, Zhong Y, Cao Y, Liu X and Huang C 2013a Curr. Appl. Phys. 1347

Zhang Y, Zhang J, Fan M, Long Y A, Zhong Y, Liu X and Huang C 2013b Bull. Mater. Sci. 36345

Zhang Y, Zhang X, Huang C and Meng C 2013c Chem. Lett. 421502

Zhao T, Liu Y and Zhu J 2005 Carbon 432907

Zhao N Q, He C N, Du X W, Shi C S, Li J J and Cui L 2006 Carbon 441859

Zhao N H, Zhang P, Yang L C, Fu L J, Wang B and Wu Y P 2009 Mater. Lett. 631955 